\title{
Trading strategies of institutional investors in a limit order book market
}

\author{
Naiwei Chen ${ }^{1,2}$ and Mingxu Peng ${ }^{1,3, a}$ \\ ${ }^{1}$ Center of Management Decision Valuation Research, Jiangxi Normal University, Nanchang, China \\ ${ }^{2}$ International Institute for Financial Research, Jiangxi Normal University, Nanchang, China \\ ${ }^{3}$ College of Finance, Jiangxi Normal University, Nanchang, China
}

\begin{abstract}
The study aims to examine the trading strategies of institutional investors in the limit order book market. The study modifies the assumptions of prior studies $[1,2]$ to match actual situations or to facilitate calculations. First, the investors' objective in the study is profit maximization rather than minimization of trading costs. Second, time is continuous rather than discrete. Third, price impact functions are non-linear and take the quadratic form that features increasing prices. Study results indicate that institutional investors adopt the increasing trading strategy if the permanent price impact dominates whereas they adopt the decreasing trading strategy if the transient price impact dominates. In addition, the average trading strategy is adopted if and only if the permanent and transient price impacts are combined in some fixed proportions.
\end{abstract}

\section{Introduction}

Most research on traditional microstructure mainly focuses on the market-maker market. However, the electronic trading system has been gradually accepted by many financial markets and has become increasingly common in real-life securities trading. Unlike the traditional dealership market, the electronic trading system is a new type of trading method in the sense that it mainly adopts the form of limit orders. Research on limit order book market can be dated back to 1960s. However, it is not until 1990s that this kind of research sees a big progress [3]. Prior studies mostly deal with the development of trading strategies and can be divided into two kinds, including studies that consider the risk of adverse selection [3-6] and those without considering such a feature [7-12].

Research on the limit order book market in China is relatively scarce. Empirical research mainly focuses on the limit order from the perspective of information asymmetry [13]. Shanghai and Shenzhen stock market and three major commodities futures market in China all adopt the limit order quotation system. Hence, it is worthwhile to study such a system in the sense that study results will provide important theoretical and empirical implications for understanding and improving the microstructure of securities market in China characterized by the limit order book quotation system.

In the limit order book market, institutional investors can easily affect the market price since their trading volume is large. Hence, it is essential and meaningful to examine the trading strategies of institutional investors and how they influence the prices and stability in the market. In fact, many studies have been devoted to this research area. For example, it is found that the average trading strategy is optimal for institutional investors from the cost minimization perspective [2]. However, it is also found that adopting the large-volume trading strategy in the beginning and the end as well as taking the average trading strategy in between is optimal based the same perspective [1]. In addition, another kind of research uses the methodology related to physical economics to study the function that deals with the impact of trading volume on prices. In particular, such research analyzes the characteristics of physical statistics for the limit order book market [13,14-20].

The study aims to examine the trading strategies of institutional investors in the limit order book market. The study modifies three assumptions of prior studies [1,2] to match actual situations or to facilitate calculations. First, the investors' objective in the study is profit maximization rather than minimization of trading costs. Second, time is continuous rather than discrete. Third, based on the research of physical economists, price impact functions are non-linear and take the quadratic form that features increasing prices.

Study results indicate that investors adopt the strategy of increasing trading volume if the permanent price impact dominates. That is, investors initially trade less to reduce the price impact and then increase trading volume gradually as time goes by. By contrast, if the transient price impact dominates, investors adopt the strategy of decreasing trading volume. That is, if large trading volume does not result in big change in long-term prices,

\footnotetext{
$\overline{\mathrm{a} C \text { Corresponding author: pengmingxu@gmail.com }}$
} 
investors will choose to engage in large-volume trading at the early stage and then decrease trading volume over time. In addition, if and only if the permanent and transient price impact functions are combined in some fixed proportions, investors will adopt the average trading strategy. Such a conclusion is inconsistent with the findings of prior studies [1,2]. However, our finding is more consistent with intuition and realities. Furthermore, the study finds that security price changes depend on the final value of securities rather than the initial value, consistent with the finding of prior research [21].

The remainder of the paper is structured as follows. The assumptions of the model are first provided in section 2. It should be noted that the study considers one-sided market only, where sellers provide limit orders and buyers provide market orders. Based on the assumptions, section 3 proposes the investors' objective function and solve for the profit maximization for institutional investors. The study solves for the objective function using dynamic programming and conduct static analysis based on the results. Section 4 concludes the paper.

\section{Hypotheses}

Limit order is an order to trade a certain number of shares at a given price or better. For the buyers, the limit orders placed below the best bid price can be considered as the total demand. For the sellers, the limit orders placed above the best ask price can be considered as the total supply. In addition, market orders will arrive continually in the limit order market, where market order is an order to trade a certain number of securities at the best bid price for sellers or at the best ask price for the buyers. Since buyers' and sellers' limit orders and their corresponding market orders are symmetric, the study considers the sellers' limit orders and the corresponding buyers' market orders to facilitate analysis.

Suppose traders need to buy some security during the continuous time period $[0, T]$ and $x(t)$ is the number of securities bought at time t. The cumulative number of securities bought by investors during the period $[t, T]$ is $Q_{t}$, which can be expressed as $Q_{t}=\int_{t}^{T} x(\tau) d \tau$. That is, $Q_{t}$ is the number of securities yet to be traded but needed to be traded by institutional investors during the period $[t, T]$. We then have $\partial Q_{t} / \partial t=-x_{t}$. After a given time $T$, institutional investors cannot and will not engage in securities trading. At the same time, suppose institutional investors are well informed such that they know that the settlement value of some security at a given time in the future is $V$. It follows that the investors 'objective is to make sure that securities are traded at prices not greater than the future settlement value (i.e., $p_{t} \leq V, t \leq T$ ) in the period $[0, T]$ and that the price at time $T$ equals the settlement value (i.e., $p_{T}=V$ ). The collection of optimal strategies for institutional investors can then be expressed as: $\varphi=\left\{(t, x(t)): t \in[0, T], \forall x(t) \geq 0, p_{T}=V\right\}$.

In addition, suppose investors are risk-neutral and trade a certain amount of securities $x(t)$ at a price $p_{t}$. The trading volume has impact on the price, which can be divided into two kinds, i.e., transient and permanent price impacts. Based on the conclusions drawn by physical economists, the price impact function is non-linear. More specifically, the larger the trading volume, the larger the price impact and the larger the variation of the price impact function, and vice versa. That is, $p^{\prime}(x)>$ $0, p^{\prime \prime}(x)>0$. Based on this, the price impact function can be expressed as $g x^{2}$, meaning that the variation of the price impact function is increasing as the trading volume increases. The permanent and transient impacts account for $\alpha$ and $1-\alpha$, respectively, where $\alpha \in[0,1]$. Accordingly, the permanent and transient price impact functions can be expressed as $\alpha g x^{2}$ and (1$\alpha) g x^{2}$ respectively. To trade securities with the amount of $x(t)$, traders are required to submit the quotations below:

$$
p+g x^{2}
$$

The transient price impact function $(1-\alpha) g x^{2}$ can only affect prices for the current period and ceases to exist in the next period. By contrast, the permanent price impact function can influence the prices in the future. Hence, the speed of price variation is $d p / d t=\alpha g x^{2}$, where $p=p_{0}$, when $t=0$ and $p=V$ when $t=T$

\section{Model and analysis}

Based on the above assumptions, the trading volume provided by institutional investors can influence trading prices. That is, there is a function where trading volume affects prices. The solution for the optimal strategy of institutional investors therefore depends on how institutional investors deal with the relationship between the price impact function and trading volume to maximize their profits. The objective function of institutional investors is assumed as follows:

$$
\begin{aligned}
& J(t, p, x)=\max _{x} E\left[\int_{0}^{T}\left[V-\left(p+g x^{2}\right)\right] x d t\right] \\
& \text { s.t. } \frac{d p}{d t}=\alpha g x, x \geq 0, p_{T}=V .
\end{aligned}
$$

Solving for the trading strategy of institutional investors yields the following:

$$
x=\frac{1}{3}\left[\sqrt{\alpha^{2} Q_{t}^{2}+\frac{3(V-p)}{g}}-\alpha Q_{t}\right]
$$

Proposition 1: In the limit order book market, if the impact of trading volume on prices satisfies equation (1) and the objective function of institutional investors satisfies equation (2), then the optimal strategy is for the trading volume to satisfy equation (3).

From equation (3) in Proposition 1, the price movement can be derived as: $p=V-g\left[2 \alpha x Q+3 x^{2}\right]$. This indicates that in a limit order book market where there are institutional investors, if the settlement value $V$ at some time in the future is known and institutional investors' objective is to maximize their returns at time $T$, then the price movement depends on the future settlement value, permanent price impact function, intended trading volume and the current trading volume of institutional 
investors. More specifically, the security price is negatively related to the permanent price impact function, current trading volume, and remaining trading volume. In addition, the security price is positively related to final settlement value. Furthermore, the security price will converge to $V$ eventually and has no relationship with the initial value.

We then conduct comparative statics for the trading strategy. Based on equation (3), the following equation is derived:

$$
\frac{\partial x}{\partial t}=\frac{(2 \alpha-3 g) x_{t}^{2}}{2 \sqrt{\alpha^{2} Q_{t}^{2}+\frac{3(V-p)}{g}}}
$$

Equation (4) describes how the trading volume of institutional investors evolves over time. There are three scenarios.

First, when $2 \alpha>3 g, \partial x / \partial t>0$, This means that when the proportion of the permanent price impact is sufficiently high, institutional investors choose the strategy of increasing trading volume. That is, if the impact of trading volume on prices is permanent, institutional investors will initially trade fewer securities to avoid excessive price increases in the future and then trade increasingly more over time. Second, when $2 \alpha<3 g, \partial x / \partial t<0$. This means that when the proportion of the permanent price impact is smaller, institutional investors choose the strategy of decreasing trading volume. That is, if the transient price impact dominates such that the impact of trading volume on prices is temporary rather than long-lasting, institutional investors will trade more securities initially and then decreases trading volume over time. Although the price can increase dramatically in response to increased trading volume, such a price increase is mostly a transient effect such that it does not take long for the price to revert to the original level. Third, when $2 \alpha=3 g, \partial x / \partial t=0$. This means that when the permanent and transient price impacts are combined in some fixed proportions, institutional investors choose the average trading strategy. That is, the average trading strategy documented in prior studies [1,2] will only be adopted by institutional investors under a particular circumstance. The average trading strategy is mere a special case among all trading strategies of institutional investors and its feasibility depends on the presence of a special market structure.

Taking the partial derivative with respective to $g$, we have:

$$
\frac{\partial x}{\partial g}=-\frac{(V-p)}{2 g^{2} \sqrt{\alpha^{2} Q_{t}^{2}+\frac{3(V-p)}{g}}} \leq 0
$$

Equation (5) indicates that the trading volume of institutional investors at time $t$ is inversely related to the coefficient of the price impact function. That is, if the coefficient of the price impact function increases, institutional investors will choose smaller trading volume at time $t$ to minimize the price impact.
Taking the partial derivative with respective to $\alpha$, we have:

$$
\frac{\partial x}{\partial \alpha}=\frac{Q_{t}}{3}\left[\frac{\alpha Q_{t}}{\sqrt{\alpha^{2} Q_{t}^{2}+\frac{3(V-p)}{g}}}-1\right]<0
$$

Equation (6) indicates that investors will trade increasingly less as the permanent price impact increases. Given constant total trading volume, investors are inclined to trade as fast as possible rather than postponing their trading. As a result, investors will surely choose to trade more initially and trade less in the future. This is because when the permanent price impact dominates and becomes increasingly large, quick transactions can avoid higher payment for future trading.

Combining equations (3) to (6), it can be inferred that in general, the impact of trading volume on prices can cause institutional investors to choose different trading strategies, further influencing the movement of security prices. In the price impact function, if the proportion of the permanent price impact $(\alpha)$ is relatively high, meaning that the majority of the price impact will continue to affect future trading, investors will surely opt for quick and large-volume transactions. By contrast, if the proportion of the permanent price impact $(\alpha)$ is relatively low, meaning that the transient price impact dominates, most price impacts will vanish quickly. Investors will surely opt to trade slowly in order not to affect future prices too much.

The permanent price impact function deals with the extent to which institutional investors disclose information to the market. Stronger permanent price impact suggests that more information contained in trading can be disclosed to the market in the form of price changes. If investors disclose more information, they should trade as soon as possible and the trading volume should be as large as possible. By contrast, the transient price impact function is like a one-time shock. It does not contain any information and prices will revert to the original level immediately after any shock. When the transient price impact dominates, investors will opt for slow trading to prevent from excessive disclosure of information, which is good for profit maximization. Such inferences concur with the conclusions of prior research [21] and the realities of securities markets. have:

Taking the partial derivative with respective to $Q$, we

$$
\frac{\partial x}{\partial Q_{t}}=\frac{\alpha}{3}\left[\frac{\alpha Q_{t}}{\sqrt{\alpha^{2} Q_{t}^{2}+\frac{3(V-p)}{g}}}-1\right]<0
$$

Equation (7) indicates that the trading volume at some point in time is positively related to the total volume that investors are willing to trade in the future. That is, if there are still many securities yet to be traded, the current trading volume has to increase.

In sum, trading strategies of institutional investors 
depend on the price impact function and the structure of permanent and transient impacts. Results are inconsistent with those of some studies [1,2]. However, results are consistent with the real situations and the finding of another study [21]. That is, if information can be hidden effectively, inside traders will not engage in large-volume trading to reveal their information. Instead, they will disclose their information gradually.

\section{Conclusions}

The paper studies the trading strategies of institutional investors in a limit order book market. We modify the assumptions and the objective function in existing literature [1,2] and use different methods to solve for optimal trading strategies.

Institutional investors have been the major traders in the securities markets. The trading strategies of institutional investors have also been the major concern of institutional investors as well as regulators, other institutions, and private investors. Based on the assumption of profit maximization, the study finds that the average trading strategy is optimal for institutional investors, concurring with the finding of prior research based on the assumption of cost minimization [2]. In behavioral finance, it is difficult to derive consistent results based on cost minimization and profit maximization $[22,23]$. However, the study shows that the average trading strategy is optimal for institutional investors based on profit maximization, concurring with the finding based on cost minimization.

\section{Appendix: Solution for Proposition 1}

Objective function:

$$
\begin{aligned}
& J(t, p, x)=\max _{x} E_{0}\left[\int_{0}^{T}\left[V-\left(p+g x^{2}\right)\right] x d t\right] \\
& \text { s.t. } \frac{d p}{d t}=\alpha g x, x \geq 0, p_{T}=V .
\end{aligned}
$$

A Hamilton function can then be generated as follows:

$$
H=(V-p) x-g x^{3}+\lambda \alpha g x^{2}
$$

Taking the partial derivative with respective to $x$, we have:

$$
\begin{gathered}
\frac{\partial H}{\partial x}=(V-p)-3 g x^{2}+2 \alpha g \lambda x=0 \\
\lambda^{\prime}=-\frac{\partial H}{\partial p}=x
\end{gathered}
$$

With $\lambda(T)=0$, we have $\lambda(t)=-Q_{t}$.

Substituting the above into equation (9), we have:

$$
\begin{aligned}
x & =\frac{\sqrt{\alpha^{2} g^{2} Q_{t}^{2}+3 g(V-p)}-\alpha g Q_{t}}{3 g} \\
& =\frac{1}{3}\left[\sqrt{\alpha^{2} Q_{t}^{2}+\frac{3(V-p)}{g}}-\alpha Q_{t}\right]
\end{aligned}
$$

Taking the partial derivatives with respective to $\alpha, g, Q_{t}$, and $t$, we have:

$$
\begin{gathered}
\frac{\partial x}{\partial \alpha}=\frac{Q_{t}}{3}\left[\frac{\alpha Q_{t}}{\left.\sqrt{\alpha^{2} Q_{t}^{2}+\frac{3(V-p)}{g}}-1\right]<0}\right. \\
\frac{\partial x}{\partial g}=-\frac{(V-p)}{2 g^{2} \sqrt{\alpha^{2} Q_{t}^{2}+\frac{3(V-p)}{g}}}<0 \\
\frac{\partial x}{\partial Q_{t}}=\frac{\alpha}{3}\left[\frac{\alpha Q_{t}}{\sqrt{\alpha^{2} Q_{t}^{2}+\frac{3(V-p)}{g}}-1}\right]<0 \\
\frac{\partial x}{\partial t}=\frac{3 g x+\alpha \sqrt{\alpha^{2} Q_{t}^{2}+\frac{3(V-p)}{g}}-2 \alpha^{2} Q_{t}}{6 \sqrt{\alpha^{2} Q_{t}^{2}+\frac{3(V-p)}{g}}} x
\end{gathered}
$$

\section{References}

1. L.R. Glosten, J Financ 49, 1127-1161 (1994)

2. B. Biais, D. Martimort, J.-C. Rochet, Econometrica 68, 799-837 (2000)

3. S. Chakravarty, C.W. Holden, J Financ Intermed 4, 213-241 (1995)

4. T. Foucault, Journal Financ Mark 2, 99-134 (1999)

5. D.J. Seppi, Rev Financ Stud 10, 103-150 (1997)

6. C.A. Parlour, D.J. Seppi, Rev Financ Stud 16, 301-343 (2003)

7. S. Viswanathan, J.J.D. Wang, J Financ Mark 5, 127-167 (2002)

8. C.A. Parlour, Rev Financ Stud 11, 789-816 (1998)

9. T. Foucault, O. Kadan, E. Kandel, Rev Financ Stud 18, 1171-1217 (2005)

10. A.A. Obizhaeva, J. Wang, J Financ Mark 16, 1-32 (2013)

11. I. Rosu, Working paper, University of Chicago (2004)

12. W.-X. Zhou, Quant Financ 12, 1253-1263 (2012)

13. R. Almgren, N. Chriss, J Risk 3, 5-40 (2001)

14. J.P. Bouchaud, M. Mézard, M. Potters, Quant Financ 2, 251-256 (2002)

15. M. Potters, J.-P. Bouchaud, Physica A 324, 133-140 (2003)

16. E. Smith, J.D. Farmer, L.S. Gillemot, S. Krishnamurthy, Quant Financ 3, 481-514 (2003)

17. H. Luckock, Quant Financ 3, 385-404 (2003)

18. P. Gopikrishnan, V. Plerou, H.E. Stanley, working paper, Massachusetts Institute of Technology, Department of Economics (2003)

19. S. Maslov, M. Mills, Physica A 299, 234-246 (2001)

20. P. Weber, B. Rosenow*, Quant Financ 5, 357-364 (2005)

21. A.S. Kyle, Econometrica, 1315-1335 (1985)

22. M. Kritzman, Financ Anal J 50, 14-18 (1994)

23. M. Kritzman, Economics and Portfolio Strategy, New York: Peter L. Bernstein, Inc. (September 1997) 